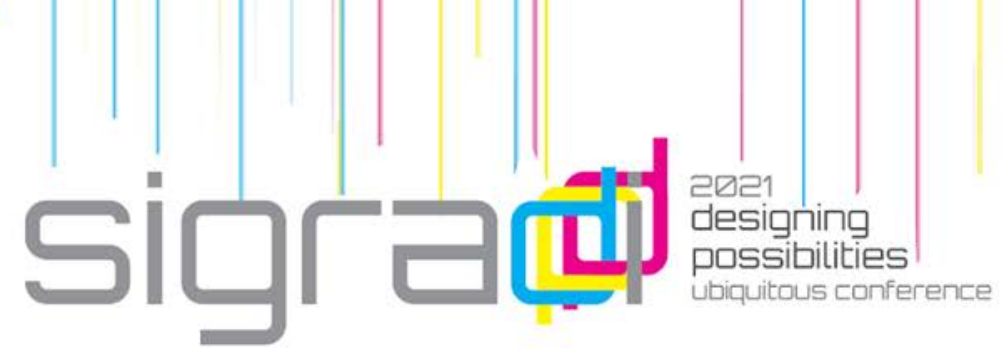

\title{
Markets Post Covid-19: Agent-Based Computational Validation Methodology For Urban Interventions On Spontaneous "Informal Street Markets" In Public Spaces
}

\author{
Arturo F. Gutiérrez ${ }^{1}$, Jeshua H. Roig ${ }^{1}$, Carlos D. Martínez ${ }^{1}$ \\ ${ }^{1}$ Escuela de Arquitectura de Lima, Perú \\ agutierrez.ealima@gmail.com \\ Iroigv@uni.pe \\ carlos.martinez18@unmsm.edu.pe
}

\begin{abstract}
The Covid-19 health crisis has turned spontaneous "informal street markets" into dangerous hotspots for the spread of Covid-19 due to the formation of crowds of people. These informal markets are due to a lack of state planning and regulation, a reality that exists throughout Latin America. This research aims to analyse these spaces through a methodology for computational validation that uses an agent-based model (ABM) for the abstraction and simulation of the displacement of people (moving agents) and their behaviour in the spatial configuration of the area (static agents), identifying an aggregated score in each simulation with the purpose of designing urban interventions that reduce the probability of forming crowds. The paper presents the proposed methodology and the ABM with a preliminary validation by simulating two spatial configurations with two hypothetical scenarios (analyses with 10 and 50 agents) and comparing their aggregated scores, showing a correlation between spatial configuration with the formation of crowds.
\end{abstract}

Keywords: Agent-based model, Spatial simulation, Stochastic model, Street market, Informal market

\section{$1 \quad$ Introducción}

La pandemia del Covid-19 ha evidenciado los problemas estructurales que afectan a los mercados peruanos, siendo el comercio informal en las calles aledañas los más complejos y difíciles de enfrentar (Concurso Mercados Post C19, 2020). La falta de planificación y la poca regulación del Estado han dado lugar a espacios caóticos, propicios para la formación de aglomeraciones de personas, convirtiéndose en peligrosos focos de contagio del SARS CoV-2 y 
sus variantes. El caso de estudio propuesto, el jirón Sebastián Barranca, aledaño al mercado 3 de Febrero, es el caso paradigmático de esta situación en el país.

En este contexto, el alcance de la investigación es desarrollar una metodología de validación computacional para propuestas de intervención urbana, con la finalidad de reducir y/o gestionar las aglomeraciones. El objetivo del presente artículo es sentar las bases de la metodología, alineando una propuesta de modelo basado en agentes (ABM), con los problemas de diseño para el caso de estudio. Partiendo desde la simulación sobre dos escenarios hipotéticos con el propósito de establecer los Índices de Aglomeración $\left(I_{A}\right)$ en respuesta a la prevención de contagios.

\section{Estado del arte}

En el urbanismo, se han utilizado los procesos estocásticos para intentar modelar sistemas complejos (Batty, 2007), bajo la premisa que en la ciudad se presentan acciones de manera aleatoria; por lo que un modelo estocástico da una gama de posibilidades, a diferencia de un modelo determinista con reglas estrictas (Shiryaev, 1996).

El avance en el poder de cómputo significó un gran empuje en la modelación e implementación de metodologías de validación computacional en los estudios urbanos, los cuales permitieron abarcar estudios de crecimiento urbano como en Batty \& Xie (1994), Clarke, Hoppen \& Gaydos (1997), He, Okada, Zhagg, Shi, Li (2008) o Al-kheder, Wang \& Shan (2013) hasta estudios de simulación del comportamiento humano para el diseño como Roetzel (2014) o Serginson et al. (2013).

Por otro lado, las aglomeraciones de personas han sido estudiadas y simuladas para garantizar seguridad (Kretz, 2007), análisis de congestión y tráfico (Hao et al., 2019) y confort (Asriana et al., 2016; Haradji, 2021), mas no como condicionantes del diseño.

Siguiendo este camino, la investigación se enfoca en elaborar una metodología y ABM de naturaleza probabilística (Helbing \& Molnar, 1995; Crooks, Castle \& Batty, 2008; Asriana and Indraprastha, 2016) identificando índices de aglomeración mediante el método Monte-Carlo (Borges et al., 2006; Shih et al., 2009) considerando aportes de estudios previos de modelación y simulación de la movilidad humana (Barbosa, Barthelemy, Ghoshal, Charlotte, Maxime, et al, 2018).

\section{3 Área de estudio}

El Jirón Sebastián Barranca en Lima es un caso paradigmático del poco o nulo control que se tiene sobre las aglomeraciones en las calles comerciales 
Personas: Agentes en movimiento que permiten la ocupación simultánea de otro agente en una misma posición, superponiéndose. Esta regla permite la identificación de las aglomeraciones.

Se considera al movimiento de los agentes como un fenómeno continuo, donde un agente solo puede moverse a los cuadrados adyacentes o puede mantener su posición. Dicha regla se complementa con las lógicas "aleatorias" (dependiente de una distribución de probabilidad).

Para el experimento se consideró el movimiento de los agentes como una distribución de probabilidad uniforme, movimiento browniano discreto, (Barbosa, Barthelemy, Ghoshal, Charlotte, Maxime, et al, 2018) la cual establece que la probabilidad de que un agente se mueva a su posición adyacente (o conserve su posición) dependerá solo del número de posiciones adyacentes a ocupar (considerando que todas son iguales). Además, el modelo considera el movimiento de las personas como un sistema dinámico estable, respecto a las diferentes configuraciones propuestas; de esta manera pequeños cambios en la distribución de los obstáculos no supondría grandes cambios en las distribuciones de probabilidad, dado que la adición y sustracción de un obstáculo en su posición solo afecta el movimiento de los agentes en cuadros cercanos a esta.

Determinado el comportamiento de los agentes para el modelo, se establece la definición del índice de aglomeraciones bajo un criterio de prevención. El objetivo de la definición es condensar la probabilidad de aglomeración en un valor numérico, dentro de una configuración de obstáculos en el espacio. Se establece la siguiente función:

$$
I_{A}(C)=\sum_{t \in T} \sum_{g \in G} e \# p(g, t)
$$

Donde IA representa el índice de aglomeración, C es la configuración específica de obstáculos en el espacio de trabajo, $T$ es el espacio de tiempo considerado en el modelo, $G$ es el conjunto de cuadrados de la grilla del espacio, $y \# p(g, t)$ es el número de personas en el cuadrado g para el momento t.

Para cada configuración de obstáculos en el espacio de trabajo se simula una cantidad "suficiente" de veces, obteniéndose un promedio del índice de aglomeración (Método Monte-Carlo). De acuerdo a las configuraciones espaciales propuestas se registra una escala de $I_{A}$ para identificar las configuraciones espaciales que ofrezcan $I_{A}$ menores para comprender las que reducen la probabilidad de contagios.

El código utilizado para la simulación del presente modelo se escribió en el lenguaje de programación multiparadigma Python 3 , ejecutándose en el entorno de trabajo interactivo Jupyter Notebook. 

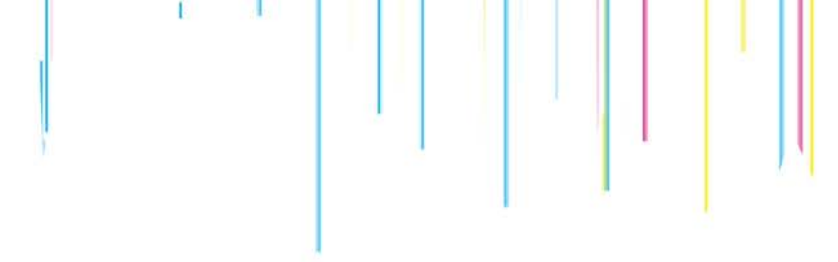

\subsection{Simulación y resultados preliminares}

Debido a la capacidad computacional disponible, se establece la simulación del $A B M$ en una cuadrícula de $10 \times 10$ unidades espaciales $(1 \mathrm{~m})$ y un lapso de tiempo de 500 unidades temporales (1s).

Como criterio, se establece que una aglomeración se identifica cuando en una misma posición (cuadrado de $01 \times 01$ ) se presentan más de un agente (persona) en un momento específico. Esta regla está en referencia al parámetro propuesto por el Ministerio de Salud del Perú de mantener un distanciamiento físico no menor a un (01) metro, como medida de prevención de contagio de covid-19 (D. S. N 094-2020-PCM, 2020).

Se establecieron dos configuraciones de obstáculos para los dos escenarios hipotéticos, denominados $\mathrm{C}_{1}$ y $\mathrm{C}_{2}$ respectivamente, en la cuadrícula de 10 × 10 unidades (Ver figura 2).

C1

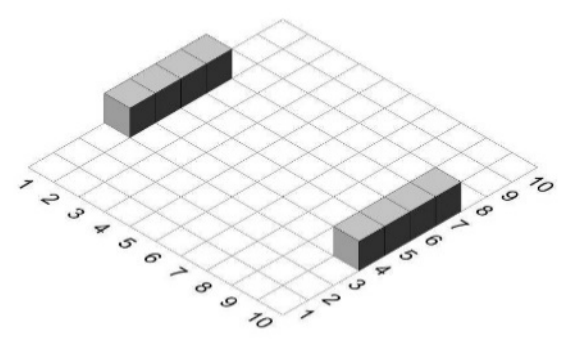

C2

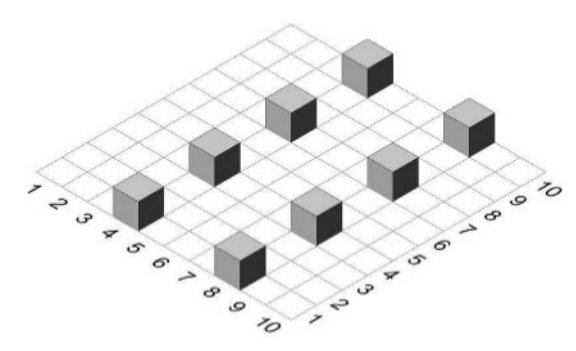

Figura 1. Configuración de agentes estáticos (obstáculos) para cada escenario en la cuadrícula de 10 x 10. Fuente: Arturo Gutiérrez, Jeshua Roig y Carlos Martinez, 2021.

Posición de obstáculos en la Configuración $\mathrm{C}_{1}$ :

$$
\# C_{1}=[[1,5],[1,6],[1,7],[1,4],[10,4],[10,5],[10,6],[10,7]]
$$

\section{Posición de obstáculos en la Configuración $C_{2}$ :}

$$
\# \mathrm{C}_{2}=[[4,1],[4,4],[4,7],[4,10],[8,1],[8,4],[8,10],[8,7]]
$$

La simulación se ejecutó con el método Monte-Carlo 50,000 veces, considerando el comportamiento de 10 y 50 personas en ambas configuraciones, obteniendo los siguientes resultados:

- Para las simulaciones con 10 agentes en movimiento (personas) se obtienen los índices de aglomeración $\left(I_{A}\right)$ de las configuraciones $C_{1}$ y $C_{2}$ de 1.57989 y 1.5797 respectivamente. Los resultados evidencian que 
las configuraciones no afectan considerablemente el índice de aglomeración cuando el número de personas es reducido.

- Para las simulaciones con 50 agentes en movimiento (personas) se obtienen los índices de aglomeración $\left(\mathrm{I}_{\mathrm{A}}\right)$ de las configuraciones $\mathrm{C}_{1}$ y $\mathrm{C}_{2}$ de 3.1499 y 3.8999 respectivamente. Los resultados evidencian que las configuraciones afectan el índice de aglomeración por el aumento de personas. Además, se observa que la configuración de obstáculos $\mathrm{C}_{2}$ genera una mayor probabilidad de formación de aglomeraciones, en comparación con la configuración $\mathrm{C}_{1}$.

Las simulaciones de los escenarios hipotéticos permiten identificar los índices de aglomeración y validar los primeros 3 pasos de la metodología, dando como resultado no sólo el mejor escenario, sino los valores numéricos que permiten posicionarlos en una escala comparativa.

Además, los resultados permiten identificar que la configuración de los agentes estáticos a ambos lados de la calle $\left(C_{1}\right)$ es mejor que la configuración de estos de manera central $\left(\mathrm{C}_{2}\right)$ en relación a un menor índice de aglomeración.

\section{Conclusiones y trabajos futuros}

Con los resultados se validan los principios generales del modelo y los primeros pasos de la metodología propuesta, determinando una relación entre la configuración espacial de los objetos y el número de personas en movimiento. Sin embargo, estos resultados son hipotéticos por lo que se requiere recopilación de data real para calibrar el modelo.

A nivel de propuesta, se elaboró un $A B M$ que contribuye al análisis cuantitativo del espacio a través de simulaciones probabilísticas que permiten categorizar propuestas de intervención bajo el criterio de reducción de aglomeraciones para prevenir contagios. Por otro lado, la investigación permite reflexionar sobre el comportamiento de las personas en un entorno geográfico específico. Se hace necesario el testeo con otros casos similares.

A nivel metodológico, la investigación propone la inclusión de herramientas de modelación matemática y validación computacional para el diseño urbano, para la simulación de escenarios hipotéticos y reales a fin de testear una gran variedad de propuestas y comprobar su eficacia.

Asimismo, la investigación permite cuestionar, reflexionar y ampliar el estudio sobre la movilidad y el diseño urbano, en donde el ABM contribuye a validar preliminarmente el comportamiento de los agentes en movimiento frente a una configuración de objetos estáticos, sirviendo de herramienta de análisis espacial y evidenciando, numéricamente, el nivel de eficiencia ante una problemática específica (formación de aglomeración de personas). Sin embargo, se evidencia la necesidad de complejizar las simulaciones incluyendo otras variables y características del espacio geográfico.

Cabe resaltar la escalabilidad del modelo y metodología propuesta en las herramientas de planificación urbana, en donde la modelación matemática y 
simulación espacial permitirían desarrollar políticas y estrategias públicas de manera más activa bajo escenarios hipotéticos y reales basados en el BIG DATA. De esta manera, se identifica el potencial de extrapolación de la metodología y modelo elaborado como herramienta de análisis para espacios comerciales, y de otros tipos, para Latinoamérica, respondiendo a las necesidades de calidad urbana y sanitaria.

Es necesario señalar que este artículo es parte de una investigación en desarrollo que plantea calibrar y validar la metodología y modelos propuestos con una recopilación de datos del área de estudio, con la finalidad de concluir en lineamientos de diseño urbano y propuestas de intervención.

Urge establecer estrategias de intervención urbana post Covid-19, desde un paradigma diferente en planificación urbana; uno adaptativo, dinámico y resiliente a las crisis; uno que emerja desde la lectura del comportamiento humano en tiempo real, es decir, desde la ciencia de datos

\section{Referencias bibliográficas}

Al-Gadhi, S. A. \& Mahmassani, H. S. (1991). Simulation of crowd behavior and movement: fundamental relations and application. Transportation Research Record, 1320, 260-268.

Aloys W.J. Borgers, I.M.E. Smeets, A.D.A.M. Kemperman, and H.J.P. Timmermans (2006). Simulation of Micro Pedestrian Behaviour in Shopping Streets. In Van Leeuwen, J.P. and H.J.P. Timmermans (eds.), Progress in Design \& Decision Support Systems in Architecture and Urban Planning (pp. 101-116). Springer.

Asriana, N. Indraprastha, A, (2016). Making Sense of Agent-Based Simulation: Developing design strategy for pedestrian-centric urban space. In S. Chien, S. Choo, M. A. Schnabel, W. Nakapan, M. J. Kim, S. Roudavski (Eds.), Proceedings of the 21st International Conference of the Association for Computer-Aided Architectural Design Research in Asia, CAADRIA (pp. 343-352). The Association for Computer-Aided Architectural Design Research in Asia.

A.W.J. Borgers, H.J.P. Timmermans (June 9-11, 2004). Simulating pedestrian route choice behavior in urban retail environments. In Walk21 - V Cities for people: the fifth international conference on walking in the 21st century. Livingstreets.

Barbosa, H. Barthelemy, M. Ghoshal, G. Charlotte, J. Maxime, L. et al. (2018). Human mobility: Models and applications. Physics Reports, Elsevier, 734, 1-74.

Batty, M. (2007). Cities and Complexity. The MIT Press.

Batty, M., \& Xie, Y. (1994). From Cells to Cities. Environment and Planning B, 31-48.

Bawden, D. (2002). Revisión de los conceptos de alfabetización informacional y alfabetización digital. Anales de Documentación, 5, 361-408. http://revistas.um.es/analesdoc/article/view/2261/2251

Concurso Mercados Post C-19. (2020). Bases. https://concursomercadospc19.com/ 
https://www.researchgate.net/publication/29799949_Pedestrian_Traffic__Simulation_and_Experiments

Manson, S., An, L., Clarke, K. C., Heppenstall, A., Koch, J., Krzyzanowski, B., Morgan, F., O'Sullivan, D., Runck, B. C., Shook, E. \& Tesfatsion, L. (2020). Methodological Issues of Spatial Agent-Based Models. Journal of Artificial Societies and Social Simulation, 23(1), 3. https://doi.org/10.18564/jasss.4174

Roetzel, A. (2015). Occupant behaviour simulation for cellular offices in early design stages: Architectural and modelling considerations. Build. Simul., 8, 211-224. https://doi.org/10.1007/s12273-014-0203-6

Rubinstein, R. Y. \& Kroese, D. P. (2017). Simulation and the Monte Carlo Method. (third ed.). John Wiley \& Sons, Inc.

Serginson, M., Mokhtar, G. \& Kelly, Graham. (2015). A Theoretical Comparison of Traditional and Integrated Project Delivery Design Processes on International BIM Competitions. International Journal of 3-D Information Modeling, 2, 52-64. https://doi.org/10.4018/ij3dim.2013100105

Shih, S.; Chen, Y; Hu, S \& Lin, C. (2009). On the simulation of pedestrian behavior: A stochastic model based on Markov chain and information space. Joining Languages, Cultures and Visions: caadFutures, 862-873.

Shyriaev, A. N. (1996). Probability. (second ed.). Springer-Verlag New York Inc.

The Guardian. (17 de mayo de 2020). 'Hubs of infection': how Covid-19 spread through Latin America's markets. Recuperado el 27 de julio de 2020 de https://www.theguardian.com/world/2020/may/17/coronavirus-latin-americamarkets-mexico-brazil-peru 